\title{
EXPERIMENTS IN ORBIT DETERMINATION USING NUMERICAL METHODS *
}

\author{
C. R. TRAAS \\ Twente University of Technology, Department of Applied Mathematics, \\ P.O. Box 217, 7500 AE Enschede, The Netherlands
}

(Received November, 1982; accepted May, 1985)

\begin{abstract}
The applicability of advanced numerical methods for the solution of the orbit determination problem is studied.

The dynamics of the observed object is written as a system of integral equations. This system is solved numerically by representing the components of the force function as linear combinations of B-splines and by applying the multigrid technique. In an outer loop the orbit determination problem is solved using Newton's method.

The method is suitable for both preliminary orbit determination and orbit improvement.
\end{abstract}

\section{Introduction}

With respect to the problem of the determination of an orbit from a given set of observations one essentially distinguishes two cases: the determination of a preliminary orbit, and the improvement of an orbit which is already approximately known.

For preliminary orbit determination at least three pairs of observations are needed. The various methods which are in use to solve this problem have in common that local approximations to the two-body motion or to the weakly perturbed two-body motion are used (see Stumpff, 1959), to suit the problem for analytical treatment as much as possible.

For orbit improvement an observational surplus is needed, and one applies a weighted least squares technique to fit a 'best' orbit (numerically integrated) to the many observations.

The purpose of the work described in the present article is to determine to what extent a number of (relatively) recent developments in the field of numerical mathematics can profitably be applied in a solution method for the orbit determination problem. In the chosen solution method the dynamics of the observed object is written as a system of integral equations. This system is solved numerically by writing the components of the force function as linear combinations of B-splines

* Most of the programing and part of the analysis was done by G. Zwier, in partial fulfilment of the requirements for obtaining the degree of master in applied mathematics at the Twente University of Technology. 
and by applying the multigrid method. In an outer loop the orbit determination problem is solved, using Newton's method.

In case of preliminary orbit determination observations are close together, and they are few in number. Then the above method can be applied in its simplest form: only two unknowns are solved and, for reasons of reduced computational complexity, a simple contraction mapping on a fixed grid replaces the multigrid method.

In case of orbit improvement the (many) observations usually extend over a longer interval of time. Then a least squares version of the method, formulated for solving six unknowns, is used and, since a contraction mapping does not converge any more, the multigrid method is fully exploited.

When considered as a method for preliminary orbit determination it can be stated that, compared with the existing, more classical methods, the present method offers complete generality. I.e. even in the case that the observed object is heavily perturbed, or in the (rare, if ever occurring) case that the observations are not really close together the preliminary orbit determination may run without difficulties. With respect to the first point, the heavily perturbed object and the difficulties associated with this situation, compare Green and Marsden, 1982, writing about comet Bowell 1980b. With respect to the second point, the observations not close together, one could think of objects which at discovery are physically close to the sun, or of minor (natural) satellites of distant planets. Of more practical importance, however, is probably the fact that the numerical methods, applied in this article to a particular problem, are likely to be more widely applicable in celestical mechanics.

\section{Orbit Determination Using Splines and the Multigrid Method}

In recent years the multigrid method has shown to be a powerful tool with respect to the numerical solution of problems which are formulated as integral equations or (partial) differential equations. In this article we demonstrate the applicability of this method for solving the orbit determination problem. The orbit determination problem is a multipoint (three at least) boundary value problem: given a sequence of pairs of observation angles $\left(\lambda_{i}, \beta_{i}\right), i=1,2, \ldots, M, M \geq 3$, measured at the times $t_{1}, \ldots, t_{M}$ by an observer on the earth, the orbit of the observed object should be determined using the fact that its motion is prescribed to satisfy the equation

$$
\ddot{\underline{r}}=\underline{F}(\underline{r}(t), t) \text {. }
$$

To conform better to the nature of the present problem, i.e., a boundary value problem, the dynamics (1) of the object is represented as an integral equation (Stumpff, 1959):

$$
\underline{r}(t)=\underline{r}_{L}(t)-\int_{t_{1}}^{t_{M}} K(s, t) \underline{E}(\underline{r}(s), s) d s
$$


in which $\underline{\underline{r}}_{L}(t)$ represents a linear, uniform motion from $\underline{r}\left(t_{1}\right)$ to $\underline{r}\left(t_{M}\right)$ :

$$
\underline{r}_{L}(t)=\underline{r}\left(t_{1}\right) \frac{t_{M}-t}{t_{M}-t_{1}}+\underline{r}\left(t_{M}\right) \frac{t-t_{1}}{t_{M}-t_{1}} \text {. }
$$

$K(s, t)$ is the kernel of the integral equation:

$$
K(s, t)= \begin{cases}\frac{\left(s-t_{1}\right)\left(t_{M}-t\right)}{t_{M}-t_{1}}, & t_{1} \leq s \leq t \\ \frac{\left(t-t_{1}\right)\left(t_{M}-s\right)}{t_{M}-t_{1}}, & t \leq s \leq t_{M}\end{cases}
$$

and $\underline{F}$ is the force function (1).

By differentiating (2) twice, it is easily verified that (2) is identical to $(1)$.

\subsection{Solution of the Integral Equation}

For solving (2), with given boundary vectors $\underline{r}\left(t_{1}\right)$ and $\underline{r}\left(t_{M}\right)$, we have chosen the following way.

Approximate $F$ as a function of $t$ by a linear combination of $B$-splines of order $k$, over an adopted grid of $\operatorname{knots}\left\{\mathrm{T}_{i}\right\}$ (de Boor, 1978):

$$
\underline{E}(\underline{r}(t), t) \simeq \underline{\tilde{F}}(t) \equiv \sum_{i} \underline{c}_{i} B_{i, k}(t)
$$

where the coefficients $\underline{C}_{i}$ are 3 -vectors.

The integral

$$
\underline{J}(t)=\int_{t_{1}}^{t_{\dot{M}}} K(s, t) \sum_{i} \underline{c}_{i} B_{i, k}(s) d s
$$

can be evaluated analytically. Let the knots be chosen between $t_{1}$ and $t_{M^{\prime}}$ with

$$
\mathrm{T}_{0}=\mathrm{t}_{1}, \quad \mathrm{~T}_{\mathrm{n}}=\mathrm{t}_{\mathrm{M}^{\prime}}
$$

giving a division of the interval $\left[t_{1}, t_{M}\right]$ into $n$ subintervals. Assign a multiplicity of $\mathrm{k}+1$ to the end-knots:

$$
\begin{aligned}
& \mathrm{T}_{0}=\mathrm{T}_{-1}=\mathrm{T}_{-2}=\ldots=\mathrm{T}_{-\mathrm{k}} \\
& \mathrm{T}_{\mathrm{n}}=\mathrm{T}_{\mathrm{n}+1}=\mathrm{T}_{\mathrm{n}+2}=\ldots=\mathrm{T}_{\mathrm{n}+\mathrm{k}} .
\end{aligned}
$$


Define $\Psi(t)$ as

$$
\Psi(t) \equiv \int_{t_{1}}^{t} \int_{t_{1}}^{s} \tilde{\underline{F}}(\xi) d \xi d s=\frac{1}{k(k+1)} \sum_{i=-k+1}^{n-1} \underline{a}_{i} B_{i}, k+2(t)
$$

with

$$
\left.\begin{array}{l}
\underline{a}_{i}=\underline{a}_{i-1}+\underline{b}_{i}\left(T_{i+k+1}-T_{i}\right), \underline{a}_{-k}=\underline{0} \\
\underline{b}_{i}=\underline{b}_{i-1}+\underline{c}_{i}\left(T_{i+k}-T_{i}\right), \quad \underline{b}_{-k}=\underline{0}
\end{array}\right\}
$$

(de Boor, 1978).

Then

$$
\underline{J}(t)=\frac{t-t_{1}}{t_{M}-t_{1}} \Psi\left(t_{M}\right)-\psi(t)
$$

in which $\Psi\left(t_{M}\right)$ can be written as

$$
\Psi\left(t_{M}\right)=\frac{a_{n-1}}{k(k+1)} .
$$

The integral equation (2) then reduces to

$$
\underline{\underline{r}}(t)=\underline{r}_{L}(t)-\frac{t-t_{1}}{t_{M}-t_{1}} \Psi\left(t_{M}\right)+\Psi(t) .
$$

Sometimes in preliminary orbit determination it is desirable to constrain the orbit to be a parabola. This can be achieved, provided the force function is taken as $\underline{F}=-\underline{r} / r^{3}$ (the gravitational constant being normalized to 1$)$, by requiring the boundary vectors $\underline{r}\left(t_{1}\right)$ and $\underline{r}\left(t_{M}\right)$ to satisfy the Euler equation (Stumpff, 1959):

$$
\left(r_{1}+r_{M}+s\right)^{3 / 2}-\left(r_{1}+r_{M}-s\right)^{3 / 2}=6\left(t_{M}-t_{1}\right) .
$$

where $r_{i}=\left|\underline{r}\left(t_{i}\right)\right|, i=1, M$, and $s=\left[r_{1}^{2}+r_{M}^{2}-2\left(\underline{r}_{1} \cdot \underline{r}_{M}\right)\right]^{1 / 2}$.

\subsubsection{Contraction Mapping}

A possible solution technique for equation (9), based upon contraction mapping (also called method of successive approximations, or Picard iteration) is as follows. 
Let $\underline{r}\left(t_{1}\right)$ and $\underline{r}\left(t_{M}\right)$ be given (possibly satisfying the parabolic constraint (10), if desired).

Assume that a vector function $\underline{r}^{(p)}$ is known, for some value of the iteration index $p$, in a set of points $t=s_{1}, s_{2}, \ldots, s_{N}$, the collocation points.

Here $s_{1}=t_{1}\left(=T_{0}\right), s_{N}=t_{M}\left(=T_{n}\right)$, and $s_{1}<s_{2}<\ldots<s_{N}$.

Furthermore

$$
\underline{\underline{r}}^{(p)}\left(s_{1}\right)=\underline{r}\left(t_{1}\right)
$$

and

$$
\underline{\underline{r}}^{(p)}\left(\mathrm{s}_{\mathrm{N}}\right)=\underline{r}\left(t_{M}\right), \underline{p}=0,1,2, \ldots
$$

Solve the vector coefficients $\left\{\underline{c}_{i}\right\}$ in (5) from the linear system

$$
\begin{aligned}
& \sum_{i=-k+1}^{n-1} \underline{c}_{i} B_{i}, k^{\left(s_{j}\right)}=\underline{E}\left(\underline{r}^{(p)}\left(s_{j}\right), s_{j}\right) \\
& j=1,2, \ldots, N, \quad N \geq n+k-1 .
\end{aligned}
$$

If $N>n+k-1$, then the solution in least squares sense can be found. Next compute the vector coefficients $\underline{b}_{i}$ and $\underline{a}_{i}$ from (7), and $\psi\left(s_{j}\right)$ from (6). Then $\underline{r}\left(s_{j}\right), j=1,2, \ldots, N$, can be computed from (9), defining the next iterate $\underline{r}^{(p+I)}$. A possibility for starting is $\underline{r}^{(0)}\left(s_{j}\right)=\underline{r}_{L}\left(s_{j}\right)$.

This successive mapping process will be a contraction indeed if the ratio $\left(t_{M}-t_{1}\right)^{2} / r_{\min }^{3}$ is small enough. Here $r_{\min }$ is the minimum distance of the object to the sun during the considered interval of time. The contraction condition is easily derived from (2), in which $-\underline{r} / \underline{r}^{3}$ is substituted for $\underline{F}$ :

$$
\underline{r}(t)=\underline{r}_{L}(t)+\int_{t_{1}}^{t_{M}} K(s, t) \frac{\underline{r}(s)}{r(s)^{3}} d s .
$$

Write this equation as

$$
\underline{\underline{r}}=\underline{\Phi}(\underline{r}) \text {. }
$$

The successive approximation errors are

$$
\underline{r}^{(p+1)}-\underline{r}=\underline{\Phi}\left(\underline{r}^{(p)}\right)-\underline{\Phi}(\underline{r}) \text {. }
$$

or

$$
\underline{\Delta}^{(p+1)}=\underline{\Phi}\left(\underline{r}+\underline{\Delta}^{(p)}\right)-\underline{\Phi}(\underline{r}) .
$$


Let $\Phi^{\prime}(\underline{r})$ be the Fréchet derivative of $\Phi(\underline{r})$. The successive mappinqs will converge (at least locally) provided that $\left\|\Phi^{+}\right\|<1$ in a neighborhood of $\underline{r}$.

In the present case:

$$
\Phi^{\prime}(\underline{\underline{r}}) \underline{\Delta}=\int_{t_{1}}^{t_{M}} K(s, t) \frac{1}{r^{3}} \cdot\left[\underline{\Delta}-3 \frac{(\underline{r}, \underline{\Delta})}{r^{2}} \underline{r}\right] d s .
$$

Now

$$
\begin{aligned}
\left\|\Phi^{\prime}(\underline{r}) \Delta\right\| \leq\left\|\int_{t_{1}}^{t_{M}} K(s, t) \frac{1}{r^{3}}|2 \Delta| d s\right\| \\
\leq \frac{2}{r_{\min }^{3}}\|\underline{\Delta}\| \frac{\operatorname{MAX} \int_{\mathrm{t}}^{t_{1}}}{t_{1}} \mathrm{k}(s, t) d s \\
=\frac{2}{r_{\min }^{3}}\|\underline{\Delta}\| \cdot \frac{1}{8}\left(t_{M}-t_{1}\right)^{2} .
\end{aligned}
$$

This implies

$$
\left\|\Phi^{\prime}(\underline{\underline{r}})\right\| \leq \frac{1}{4} \frac{\left(t_{M}-t_{1}\right)^{2}}{r_{\min }^{3}},
$$

which value should be less than 1. The convergence is the faster the smaller $\left\|\Phi^{\prime}(\underline{r})\right\|$ is.

It is of importance to note that the matrix of B-splines values in (11) is always the same for fixed point sets $\left\{s_{j}\right\}$ and $\left\{T_{i}\right\}$. Therefore when changing $\underline{r}^{(p)}$, the LU decomposition of this matrix remains unaltered.

\subsubsection{Multigrid Method}

If the contraction mapping converges slowly, or converges not at all, e.g. in case of a relatively long time interval, then the multigrid method may produce a solution in an efficient way. The principle is to solve (9) on a course collocation grid in a direct way, e.g. by using a (quasi-) Newton method, and, next, to improve the solution by applying a defect correction process on a sequence of finer grids. Simultaneously relaxation sweeps are performed using the contraction mapping operator (McCormick, 1982; Hemker, 1981; Hackbusch, 1982). We here describe the process briefly for a sequence of 2 grids. More details and an illustrative example can be found in the appendix.

Let (9) symbolically be written as

$$
\underline{r}+K(\underline{r})=\underline{r}_{L^{\prime}}
$$


and let superscripts (f) and (c) denote 'fine grid' and 'course grid', respectively. To find the solution $\underline{r}^{(f)}$, the following steps must be performed:

(i) Solve $\underline{r}^{(c)}+K\left(\underline{r}^{(C)}\right)=R \underline{r}_{L}^{(f)}$ where $R$ is the restriction

(ii) Prolongate the result to the finer grid:

$$
\underline{r}^{(f)}=\underline{P}^{(c)}
$$

(iii) Smooth the prolongated result by means of $\mathrm{n}_{\mathrm{s}}$ relaxation steps $\left(n_{s} \geq 1\right):$

$$
\begin{aligned}
& \underline{\xi}_{0}^{(f)}:=\underline{\underline{r}}^{(f)} \\
& \underline{\xi}_{j}^{(E)}:=\underline{r}_{L}^{(f)}-K\left(\underline{\xi}_{j-1}^{(f)}\right), j=I, \ldots, n_{s} \\
& \text { and put } \hat{r}^{(f)}=\underline{\xi}_{n}^{(f)}
\end{aligned}
$$

These three steps are preparatory.

The actual iterative process occurs in the following steps.

Let $\underline{r}_{0}^{(f)}$ be an estimate to the solution of (9) on the fine grid, e.g.

$\underline{r}_{0}^{(f)}=\underline{\hat{x}}^{(f)}$. Then, starting with the iteration index $p=0$ :

(iv) Compute $\underline{v}_{p}^{(f)}=\underline{\underline{r}}_{\underline{p}}^{(f)}+K\left(\underline{\underline{r}}_{\underline{p}}^{(f)}\right.$ )

(v) Solve $\underline{r}_{\underline{p}+1}^{(c)}+K\left(\underline{r}_{\underline{p}+1}^{(c)}\right)=\operatorname{Rv}_{p}^{(f)}$

(i.e. solve the course grid problem with a modified right-hand member)

(vi) Prolongate the result to the finer grid:

$$
\underline{r}_{p+1}^{(f)}=P \underline{p}_{p+1}^{(c)}
$$

(vii) Smooth the prolongated result by means of $\mathrm{n}_{\mathrm{s}}$ relaxation steps $\left(n_{s} \geq 1\right):$

$$
\begin{aligned}
& \underline{\xi}_{0}^{(f)}:=\underline{r}_{p+1}^{(f)} \\
& \underline{\xi}_{j}^{(f)}:=\underline{v}_{p}^{(f)}-K\left(\underline{\xi}_{j-1}^{(f)}\right), j=1, \ldots, n_{s} \\
& \text { and put } \underline{\underline{r}}_{p+1}^{(f)}=\underline{\xi}_{n}^{(f)}
\end{aligned}
$$


(viii) Correct the defect:

$$
\underline{r}_{\underline{p}+1}^{(f)}=\underline{r}_{p}^{(f)}-\left(\bar{r}_{\underline{p}+1}^{(f)}-\underline{\hat{r}}^{(f)}\right) .
$$

As this point the loop can be exit, or $p:=p+1$, go to (iv). The result of step (viii) should converge to the solution of the problem. Actual (direct) solution only occurs on the course grid (one time in step ( $i)$, and next in step ( $v$ ) in the loop), e.g. by Newton's method.

It may be clear that, while for obtaining the solution on the fine grid the problem must repeatedly be solved on the course grid, the solutions on the course grid may be obtained, in curn, by solving on a still courser grid, etc., resulting in a nested application of the above solution method. Ultimately, the problem is solved directly on the adopted coursest grid only, which may be an easy process due to the (relatively) low number of grid points involved.

The multigrid process, presented above, is one of the possible variants of the method. The multigrid process may also be based on the dual defect correction process. Also mixed formulations are possible (Hemker, 1981). Finally the basic solution process can be changed, in the sense that the solution method to the boundary value problem is formulated as a Newton process on the finest grid. The Newton corrections, however, are computed by means of the multigrid method, which then can be reformulated for a linear operator. Advantage is that the multigrid process is performed entirely on the level of corrections. The computation as a whole, however, is more laborious.

\subsection{Solution of the Orbit Determination Problem}

The solution of the integral equation is a subroutine in the orbit determination program. The main problem is the solution of a system of $2 \mathrm{M}$ nonlinear equations of the type

$$
\left\{\begin{array}{c}
z_{i}-z_{i}-\sin \beta_{i} \cdot\left[\left(x_{i}-x_{i}\right)^{2}+\left(\underline{y}_{i}-Y_{i}\right)^{2}+\left(z_{i}-z_{i}\right)^{2}\right]^{\frac{1}{2}}=0 \\
x_{i}-x_{i}-\cos \lambda_{i} \cdot\left[\left(x_{i}-x_{i}\right)^{2}+\left(\underline{y}_{i}-Y_{i}\right)^{2}\right]^{\frac{1}{4}}=0 \\
(i=1,2, \ldots, M)
\end{array}\right.
$$

where $M$ is the number of observation times, $M \geq 3$, and where

$\beta_{i}, \lambda_{i}$

$x_{i}, y_{i}, z_{i}$

$X_{i}, Y_{i}, Z_{i}$ are observation angles at the time $t_{i}$ ' are coordinates of the observed object in a sun-centered Cartesian system, at the time $t_{i}$, are coordinates of the observer in the same system, at the time $t_{i}$.

Compare Figure 1, in which the situation is sketched for $M=3$, the minimum number of observation times. 


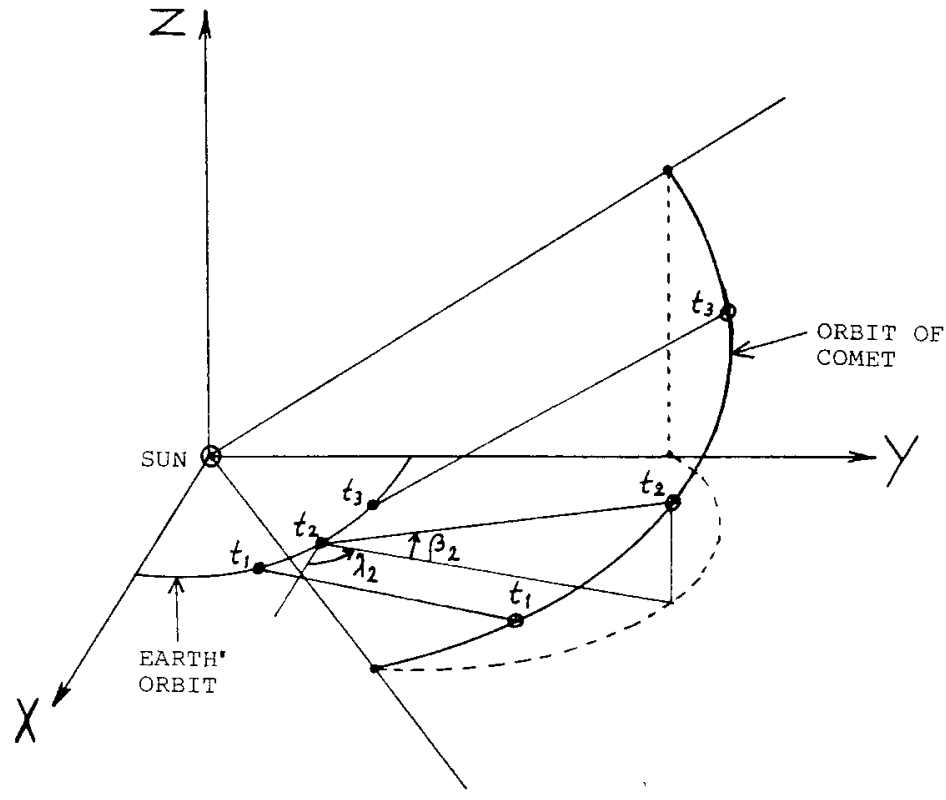

Fig. 1. General configuration.

In (12) the coordinates $x_{i}, y_{i}, z_{i}(i=1,2, \ldots, M)$ are $3 M$ unknowns. The successive positions of the observer are assumed to be known. Using an approximate solution to (2), obtained along the lines described in section 2.1, it is possible to express $x_{i}, y_{i}$ and $z_{i}(i=2,3, \ldots, M-1)$ in terms of $x_{1}, y_{1}, z_{1}, x_{M}, y_{M}$ and $z_{M}$, resulting in only six unknowns. Then (12) can be solved for these six coordinates using standard iterative methods. For $M>3$ the solution in least squares sense can be found. We have used a quasi-Newton method for this purpose.

In case of preliminary orbit determination from three observations, the number of unknowns can be reduced to two only. Suitable for this purpose are the distances from observer to object at the times $t_{1}$ and $t_{3}$. From estimates of these two distances the coordinates $x_{1}, y_{1}, z_{1}, x_{3}$, $\underline{y}_{3}, z_{3}$ can be computed with the help of the observation angles $\beta_{1}, \lambda_{1}$, $\beta_{3}, \lambda_{3}$. Then $x_{2}, y_{2}$ and $z_{2}$ can be computed by solving (2). Newton's method is used to vary both distances such, that (12) will be satisfied for $i=2$.

If one wishes to constrain the orbir to be a parabola, the Newton corrections must be projected onto the 5-dimensional manifold which is defined by the parabolic constraint (10), or, in case of two unknowns, onto the 1-dimensional manifold which is defined by (12) for $i=1$ and $i=M=3$, and by (10). The algorithms, to be used for these operations, 
are well-known from the many textbooks on constrained optimization (e.g. Himmelblau, 1972).

Once the solution has been found, the velocity of the observed object at an arbitrary time $t \in\left[t_{1}, t_{M}\right]$ can easily and accurately be found from the equation

$$
\underline{\underline{r}}(t)=\frac{\underline{\underline{r}}\left(t_{M}\right)-\underline{r}\left(t_{1}\right)}{{ }_{{ }}-t_{1}}-\int_{\tau_{1}}^{t_{M}} k(s, t) \underline{E}(\underline{r}(s), s) d s
$$

in which

$$
k(s, t)=\left\{\begin{array}{l}
\frac{t_{1}-s}{t_{M}-t_{1}}, t_{1} \leq s \leq t \\
\frac{t_{M}-s}{t_{M}-t_{1}}, t \leq s \leq t_{M}
\end{array}\right.
$$

In approximation this can be written as

$$
\underline{\underline{r}}(t)=\frac{\underline{r}\left(t_{M}\right)-\underline{r}\left(t_{1}\right)}{t_{M}-t_{1}}-\frac{\psi\left(t_{M}\right)}{t_{M}-t_{1}}+\int_{t_{1}}^{t} \underline{\tilde{F}}(s) d s
$$

in which the integral is found from

$$
\int_{t_{1}}^{t} \underline{\tilde{F}}(s) d s=\frac{1}{k} \sum_{i=-k+1}^{n-1} \underline{b}_{i} B_{i}, k+1(t),
$$

while the $\underline{b}_{i}$ are found from (7).

Due to the accuracy of this process reliable osculating elements can be computed, and reliable initial conditions can be found for numerical integration of the future orbit. This is an important reason for using the integral equation formulation for the boundary value problem (rather than applying a finite difference method directly to (1)).

\section{Numerical Results}

The described method of orbit determination has been tested for a few simulated examples, two of which are presented in this section. The configuration for the first testcase is sketched in Figure 2. This testcase does not represent a practical situation, but it illustrates the effectiveness of the used numerical techniques very well. The plane of the earth' orbit coincides with the $x, y$-plane. Parameter values of the orbit of the observed object are 


$$
\begin{aligned}
& a=0.34 \mathrm{AE} \\
& \mathrm{e}=0.53 \\
& i=14^{\circ} \\
& \Omega=202^{\circ}
\end{aligned}
$$

Observation times are: $t_{1}=0, t_{2}=0.064$ and $t_{3}=0.127$ years (thus $\mathrm{M}=3$ ).

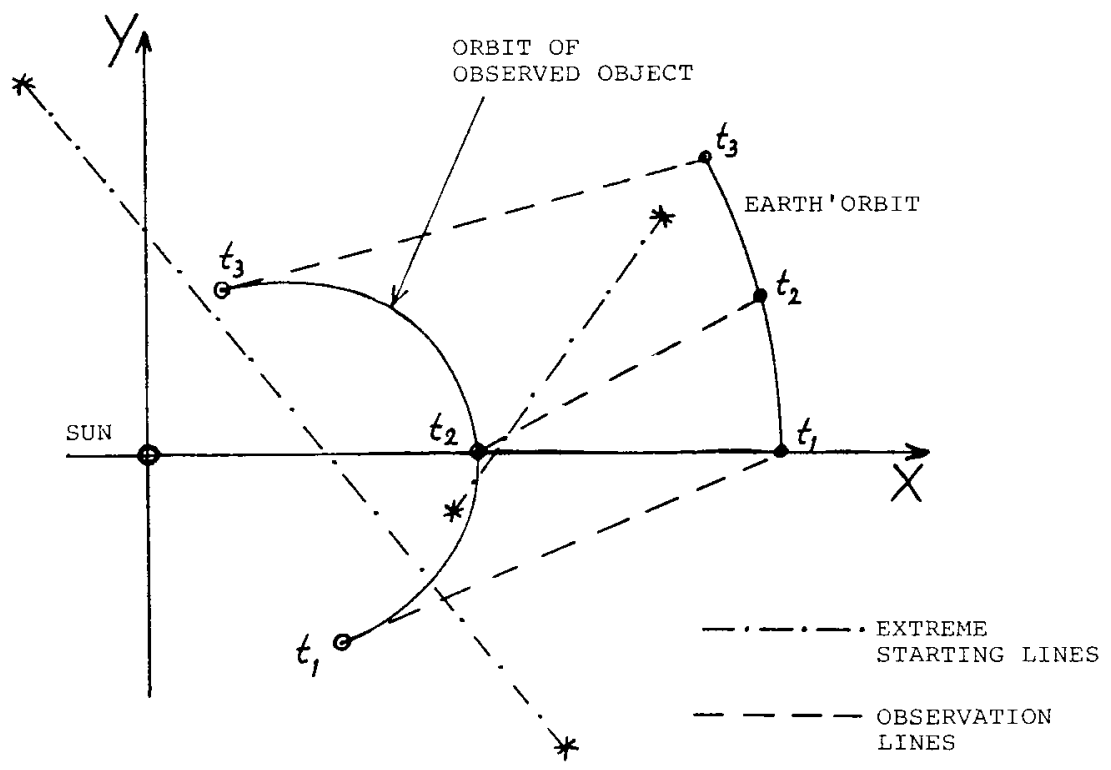

Fig. 2. Projection of simulated orbit on the plane of the ecliptic (first testcase).

For solution of the integral equation a three-level multigrid algorithm has been applied, using grids consisting of 8,16 and 32 intervals, respectively. Cubic splines have been used to approximate the components of the force function. Each multigrid $w$-cycle iteration requires about 150 function evaluations (a function evaluation should be understood as an evaluation of the force vector function $\underline{F}$ ). where on the coursest grid a Newton process is applied. The total effort required to solve the problem strongly depends on the initial estimate. For the latter a wide variety of conditions were chosen. Extreme choices are indicated in Figure 2. For an average set of initial conditions the computational effort is roughly as follows. To obtain a result with a relative error of $10^{-6}$, about six quasi-Newton steps are needed (in the outer loop). on the average one quasi-Newton step (keeping the Jacobian matrix constant, occasionally) requires four calls of the multigrid process. one complete multigrid process consists of two to five W-cycles, dependent on the initial estimate and the required local accuracy (the 
latter in view of the progressing outer loop iteration). This results in a total of about 12000 function evaluations for solution of one orbit determination problem of the type given in Figure 2, and with practically no a-priori information about the orbit. For the two extreme initial conditions given in Figure 2 about 16000 function evaluations are needed. Convergence problems only occurred with starting lines located behind the sun, and with starting lines very close to the earth' orbit.

The configuration for the second testcase is sketched in Figure 3. Here only the multigrid process for solving the boundary value problem has been tested. Parameter values of the orbit are
$a=1.51$
$\mathrm{e}=0.60$
$i=2.3^{\circ}$
$\Omega=270^{\circ}$

And the time between the given boundary points is 1.55 years.

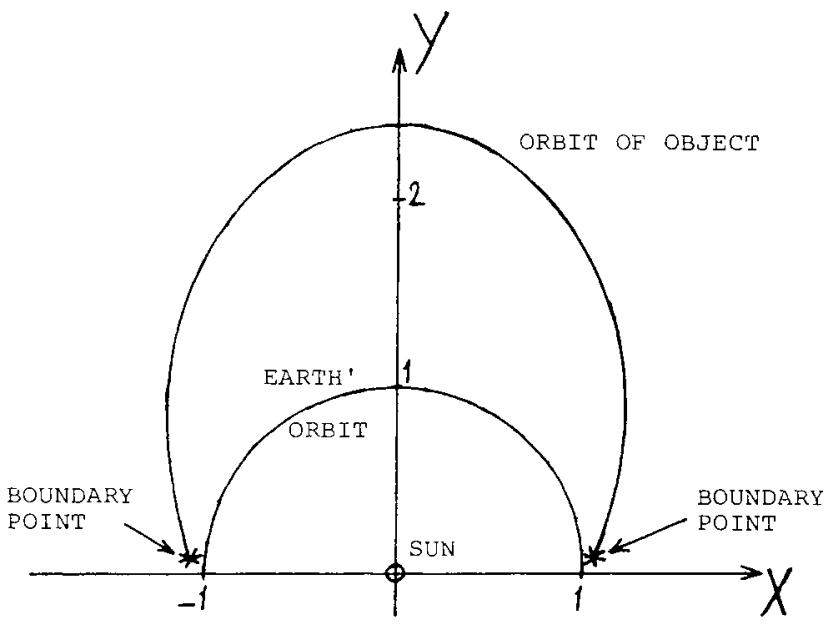

Fig. 3. Projection of simulated orbit in plane of the ecliptic (second testcase).

Applying again the three-level multigrid algorithm with grids consisting of 8,16 and 32 intervals, respectively, and starting from a straightline initial estimate, the final result is obtained with a relative error of $10^{-7}$ using about 600 function evaluations. In the latter testcase the Picard iteration process is divergent.

\section{Conclusions}

The general orbit determination problem can be solved effectively in a direct way, using advanced numerical methods.

With B-splines, a flexible function approximation is possible by choice of knot locations. Even locally deviating behaviour of a function (in the present context: severe perturbations in close encounter) can be fitted accurately. 
The multigrid method appears to be an effective method for solving the orbital boundary value problem. Using this technique, the direct solution of the boundary value problem (e.g. by Newton's method) is required on a course grid only. On finer grids accurate solutions are obtained by a defect correction process.

Using the number of function evaluations as a measure for the computational effort required, it appears that with careful implementation the multigrid process in the present application may be 10 to $15 \%$ more efficient than direct application of Newton's method on the finest grid (inner loop).

For observations extending over a short interval of time, direct application of Picard iterations for solving the boundary value problem is by far the most efficient.

\section{Acknowledgement}

I am grateful to Dr D. Dijkstra, my colleague at the Twente University, and to Dr Ir H. Schippers, of the National Aerospace Laboratory. Amsterdam, for their valuable support regarding this study.

\section{Appendix The Multigrid Method}

In this appendix a condensed exposition of the multigrid method is given, together with a simple illustrative example.

\section{Theoretical background}

Let a nonlinear operator equation

$$
\mathrm{Au}=\mathrm{f}
$$

be given. Let $B$ be an approximate inverse to the operator $A, i . e$. $B \approx A^{-1}$.

A solution to (1) may be obtained by applying the following iterative process, a defect correction process:

$$
u_{k+1}=u_{k}-\left(B A u_{k}-\hat{u}\right) \text {. }
$$

where $\mathrm{k}$ is the iteration index, $\mathrm{k}=0,1,2, \ldots ; \hat{\mathrm{u}}=\mathrm{Bf}$ is the approximate solution generated by $\mathrm{B}$, and $\mathrm{u}_{0}$ is a first estimate to the solution (e.g. $u_{0}=\hat{u}$, or better). If (2) converges to the correct solution of (1), then $\mathrm{Au}_{\mathrm{k}} \rightarrow \mathrm{E}$, and $\mathrm{BAu_{k }} \rightarrow \hat{\mathrm{u}}$. The class of Newton processes is contained in (2).

Let a discretized version of (1) be written as

$$
A^{(f)}(f)=f^{(f)} \text {, }
$$

where the superscript (f) denotes 'fine grid'. The functions $u$ and $f$ then are grid functions. In the multigrid method an approximate inverse to $A^{(f)}$ is defined by introducing courser grids. 
Let a discretization of (1) on a course grid be written as

$$
A^{(c)}(c)=f^{(C)}
$$

and assume that (4) can be solved (either directly, or by again introducing courser grids).

In the multigrid technique relations are introduced between the functions on the different grids by means of special operators: the prolongation operator $\mathrm{P}$ and the restriction operator $\mathrm{R}$. The function $\mathrm{PW}_{\mathrm{W}}(\mathrm{C})$ is the grid function on the fine grid, corresponding with the course grid function $w^{(c)}$. The function $R w(f)$ is the course grid function, corresponding with the fine grid function $w(f)$. Usually, the operator $\mathrm{P}$ respresents some incerpolation process, and the operator R represents some averaging process.

Turning back to (4), which can be solved giving

$$
u^{(C)}=A^{(C)^{-1}} f^{(C)} \text {. }
$$

where $f^{(C)}=R^{(f)}$, the multigrid method proceeds with prolongating $(c)$ to the fine grid:

$$
u^{(f)}=\mathrm{Pu}^{(\mathrm{c})} \text {. }
$$

Consider this result as an approximate solution to (3), i.e. define the approximate inverse operator $B$ as

$$
B=P A(C)^{-1} R \text {. }
$$

Then the iterative process (2) can be rewritten as

$$
u_{k+1}^{(f)}=u_{k}^{(f)}-\left(P A(C)^{-1} R A^{(f)} u_{k}^{(f)}-\hat{u}^{(f)}\right)
$$

with $\hat{u}(f)=P A(c)^{-1} R^{(f)}$.

Let (8) converge to a grid function $u^{(f)}$. Then

$$
B A^{(f)} u^{(f)}=B f^{(E)} \text {. }
$$

since the correction tends to zero.

However, the operator B, defined in the above way, is not injective (different originals may give the same image, under the mapping $B$ ). Therefore, from (9) it can not be concluded that

$A^{(f)} u^{(f)}=f^{(f)}$, and thus is $u^{(f)}$ not necessarily a solution to (3). The elements $w^{(f)}$, which satisfy $B w^{(f)}=B v^{(f)}$ for given (smooth) $v^{(f)}$, are usually highly oscillatory for $w^{(f)} \neq v^{(f)}$. Therefore these elements can be eliminated by applying a smoothing process over the fine grid, each time a prolongation has been performed. The smoothing process is a relaxation on the basis of the fine grid operator $A(f)$. 


\section{A concrete Example}

We illustrate the multigrid process numerically with the help of a simple problem. Solve $u(t)$ from

$$
\frac{d^{2} u}{d t^{2}}=e^{u}, u(-1)=u(1)=0 .
$$

\subsection{Analysis}

The problem can be rewritten as

$$
u(t)=-\int_{-1}^{1} k(t, s) e^{u(s)} d s
$$

with

$$
K(t, s)=\left\{\begin{array}{l}
\frac{1}{2}(1+s)(1-t), s \leq t \\
\frac{1}{2}(1-s)(1+t), s \geq t .
\end{array}\right.
$$

We adopt three grids.

The first (= coursest) grid consists of two steps, [-1,0] and $[0,1]$. The second grid has four (equal) steps, and the third (= finest) grid has eight (equal) steps.

The operator equation, corresponding to (1), is here

$$
A u(t) \equiv u(t)+\int_{-1}^{1} k(t, s) e^{u(s)} d s=0
$$

$t \in[-1,1]$.

Thus $f=0$ in this problem.

In discretized form this becomes, on the first grid:

$$
u(0)+\frac{1}{2} e^{u(0)}=0
$$

which equation can be obtained by applying the trapezoidal rule (2 steps) to the integral in (11).

on the second grid, the discretized version reads:

$$
\left[\begin{array}{l}
u\left(-\frac{1}{2}\right) \\
u(0) \\
u\left(\frac{1}{2}\right)
\end{array}\right]+\frac{1}{16}\left[\begin{array}{lll}
3 & 2 & 1 \\
2 & 4 & 2 \\
1 & 2 & 3
\end{array}\right]\left[\begin{array}{l}
e^{u\left(-\frac{1}{2}\right)} \\
e^{u(0)} \\
e^{u\left(\frac{1}{2}\right)}
\end{array}\right]=0
$$


obtained again by applying the trapezoidal rule (4 steps). on the third grid, finally, a similar system can be derived, this time, however, with 7 -element vectors, and a matrix of size $[7 \star 7]$.

\subsection{Numerical Results}

Solving (12) with Newton's method, gives ${ }^{(1)}(0)=-0.351734$. The upper index labels the grid. Prolongation is done by copying the grid values of the courser grid, and by interpolating linearly between the grid points of the courser grid. For the transfer from first to second grid this here gives:

$$
u^{(2)}=\left[\begin{array}{c}
-0.175867 \\
-0.351734 \\
-0.175867
\end{array}\right] \text {. }
$$

Smoothing on the second grid is done by performing one relaxation step:

$$
u^{(2)}:=-\frac{1}{16} M\left[\begin{array}{l}
\exp \left[u^{(2)}(-0.5)\right] \\
\exp \left[u^{(2)}(0)\right. \\
\exp \left[u^{(2)}(0.5)\right]
\end{array}\right]
$$

where $M$ is the matrix defined in (13). For such a step of Gauss-Seidel type the result is here:

$$
\hat{\mathrm{u}}^{(2)}=\left[\begin{array}{c}
-0.297616 \\
-0.373531 \\
-0.289711
\end{array}\right] \text {. }
$$

This result corresponds with the function which is written as $\hat{u}$ in (2), on the second grid. We proceed with the defect correction on the second grid, and we therefore adopt a first estimate, which here is chosen as $u_{0}^{(2)}=\hat{u}^{(2)}$. The lower index is the iteration index.

Computation of $\mathrm{A}^{(2)} \mathrm{u}_{0}^{(2)}$ (i.e. the vector value of the left-hand side of (13)) gives:

$$
f_{0}^{(2)}=\left[\begin{array}{l}
-0.025563 \\
-0.015073 \\
-0.016922
\end{array}\right] \text {. }
$$

Next, the equation $A^{(2)} u^{(2)}=f_{0}^{(2)}$ must be solved, using the approximate inverse to $A^{(2)}$ which also was used to compute $\hat{u}^{(2)}$. This proceeds in the same way as described above, apart from the fact that (12) and (13) 
now possess nonzero right-hand members. The restriction operation, which is required in this process to obtain the course grid right-hand member, is done by weighted averaging of the values at three consecutive grid points, with weights $1 / 4,1 / 2$ and $1 / 4$, respectively. There results a Newton solution $u^{(1)}(0)=-0.365190$. Prolongation to the second grid and one relaxation step on this grid leads to the vector

$$
\bar{u}_{1}^{(2)}=\left[\begin{array}{l}
-0.320598 \\
-0.383442 \\
-0.303675
\end{array}\right]
$$

and defect correction: $u_{1}^{(2)}=u_{0}^{(2)}-\left(\bar{u}_{1}^{(2)}-\hat{u}^{(2)}\right)$ gives

$$
u_{1}^{(2)}=\left[\begin{array}{c}
-0.274634 \\
-0.363621 \\
-0.275747
\end{array}\right] \text {. }
$$

This result is to be considered as a course-qrid solution relative to the third grid. Prolongation to the third grid, and one relaxation step on this grid results in

$$
\hat{u}^{(3)}=\left[\begin{array}{c}
-0.169093 \\
-0.282426 \\
-0.349300 \\
-0.369277 \\
-0.347971 \\
-0.280173 \\
-0.167155
\end{array}\right]
$$

which again corresponds wiht $\hat{\mathrm{u}}$ in (2), this time, however, on the third grid. We adopt as a first estimate on the third grid:

$$
u_{0}^{(3)}=\hat{u}^{(3)} \text {. }
$$

With this vector, the vector value of $A^{(3)} u_{0}^{(3)}$ can be computed, giving $f_{0}^{(3)}$. The next step is to solve $A^{(3)} u_{0}^{(3)}=f_{0}^{(3)}$ using the approximate inverse to $A^{(3)}$. This problem is reduced to solving, on the second grid, the equation:

$$
A^{(2)} u^{(2)}=\operatorname{Rf}_{0}^{(3)}
$$


which problem in turn is reduced to solving on the first grid, giving a new $\hat{u}^{(2)}$ and a new $\bar{u}_{1}^{(2)}$, and the result $u_{1}^{(2)}$ after defect correction:

$$
\hat{u}^{(2)}=\left[\begin{array}{l}
-0.301222 \\
-0.376478 \\
-0.291084
\end{array}\right], \quad u_{1}^{(2)}=\left[\begin{array}{l}
-0.277925 \\
-0.366550 \\
-0.277143
\end{array}\right] \text {. }
$$

Prolongation to the third grid, one relaxation step on this grid and defect correction gives:

$$
\bar{u}_{1}(3)=\left[\begin{array}{l}
-0.172145 \\
-0.285585 \\
-0.353454 \\
-0.371672 \\
-0.350766 \\
-0.281029 \\
-0.168212
\end{array}\right], u_{1}^{(3)}=\left[\begin{array}{l}
-0.166040 \\
-0.279268 \\
-0.345145 \\
-0.366882 \\
-0.345175 \\
-0.279317 \\
-0.166098
\end{array}\right]
$$

The iteration proceeds, starting with the computation of $A^{(3)} u_{1}^{(3)}$, etc., until the absolute corrections on the third grid are small enough. We find

$$
u_{3}^{(3)}=\left[\begin{array}{c}
-0.166113 \\
-0.279291 \\
-0.345200 \\
-0.366853 \\
-0.345200 \\
-0.279291 \\
-0.166113
\end{array}\right]
$$

which is, as solution to the discretized equation on the finest grid, correct in all digits.

The order in which the grids are called in the above example is such that one speaks about a multigrid W-cycle. It is one of the possible variants (see e.g. the first introductory paper in Hackbusch, 1982).

\section{References}

de Boor, C.: 1978, A Practical Guide to Splines, Springer Verlag. Green, D.W.E. and Marsden, B.G.: 1982, A Comet in a Million, Sky and Telescope, April, p. 366. 
Hackbusch, W.: 1982, Multigrid Methods, Lecture Notes in Mathematics 960, Springer verlag.

Hemker, P.W.: 1981. Introduction to MuItigrid Methods, Nieuw Archief voor Wiskunde (3), XXIX, pp. 71-101; Stichting Mathematisch Centrum, Amsterdam, the Netherlands.

Himmelblal,, D.M.: 1972, Applied Nonlinear Programming, McGraw-Hill Book Company.

MCCormick, S.F.: 1982, SIAM J. Num. Anal. 19, 548-560.

Stumpff, K.: 1959, Himmelsmechanik I, VEB Deutscher Verlag der Wissenschaften. 